\title{
Decaying Dark Matter in the Supersymmetric Standard Model with Freeze-in and Seesaw mechanims
}

\author{
Zhaofeng Kang ${ }^{1}$ and Tianjun $\mathrm{Li}^{1,2}$ \\ ${ }^{1}$ Key Laboratory of Frontiers in Theoretical Physics, Institute of Theoretical Physics, \\ Chinese Academy of Sciences, Beijing 100190, P. R. China \\ ${ }^{2}$ George P. and Cynthia W. Mitchell Institute for Fundamental Physics, \\ Texas A\&M University, College Station, TX 77843, USA
}

(Dated: October 30, 2018)

\begin{abstract}
Inspired by the decaying dark matter (DM) which can explain cosmic ray anomalies naturally, we consider the supersymmetric Standard Model with three right-handed neutrinos (RHNs) and $R$-parity, and introduce a TeV-scale DM sector with two fields $\phi_{1,2}$ and a $Z_{3}$ discrete symmetry. The DM sector only interacts with the RHNs via a very heavy field exchange and then we can explain the cosmic ray anomalies. With the second right-handed neutrino $N_{2}$ dominant seesaw mechanism at the low scale around $10^{4} \mathrm{GeV}$, we show that $\phi_{1,2}$ can obtain the vacuum expectation values around the $\mathrm{TeV}$ scale, and then the lightest state from $\phi_{1,2}$ is the decay DM with lifetime around $\sim 10^{26} \mathrm{~s}$. In particular, the DM very long lifetime is related to the tiny neutrino masses, and the dominant DM decay channels to $\mu$ and $\tau$ are related to the approximate $\mu-\tau$ symmetry. Furthermore, the correct DM relic density can be obtained via the freeze-in mechanism, the smallscale problem for power spectrum can be solved due to the decays of the $R$-parity odd meta-stable states in the DM sector, and the baryon asymmetry can be generated via the soft leptogensis.

PACS numbers: 12.60.Jv, 14.70.Pw, 95.35.+d
\end{abstract}




\section{INTRODUCTION AND MOTIVATION}

The cosmic ray anomalies observed by PAMELA and Fermi-LAT [1, 2] strongly indicated that the dark matter (DM) particles annihilate or decay dominantly into the leptons. Although the large annihilation cross sections can be realized via the Sommerfield enhancement or Breit-Wigner mechanism [3], the HESS obervation [4, 5] of the Galactic center gamma rays gives strong constraints on the annihilation DM scenario [6]. The decaying DM [7 [9] with a lifetime at the order $\mathcal{O}\left(10^{26}\right) s$ is another elegant way to explain the cosmic ray anomalies. In particular, the contraints from the Galactic center gamma rays are much weaker [6]. However, the ultimate long lifetime of decaying DM becomes a non-trivial problem since the symmetry, which makes the DM stable, must be broken tinily.

Supersymmetry naturally solve the gauge hiearchy problem in the Standard Model (SM). Gauge coupling unification in the Minimal Supersymmetric Standard Model (MSSM) implies the Grand Unified Theories (GUTs) at the GUT scale $M_{G U T}$ around $2 \times 10^{16} \mathrm{GeV}$. Thus, the DM decays via the dimension-six operators suppressed by the GUT scale is a rather simple solution to the long lifetime of decaying DM, and it may provide a way to probe the GUT scale physics [8]. Another problem in the decaying DM is how to understand the DM dominant leptonic decays, especially to the $\mu$ and $\tau$ final states. Without automatically kinematical suppressions like the annihilation models [3], one has to employ some special symmetry so that the DM interacts strongly with the second or third families of the charged leptons, for instance, flavor Froggat-Nielson symmetry [10].

Because of the DM leptonic decays, one may conjecture that the DM sector only interacts with the lepton sector [11]. Note that the neutrino masses are very tiny, we can parametrize the small couplings for the DM decay as the ratio between the light neutrino mass $m_{\nu} \sim 10^{-11}$ $\mathrm{GeV}$ and the GUT scale

$$
\lambda \sim \frac{m_{\nu}}{M_{G U T}} \sim 10^{-27} .
$$

Interestingly, this is the typical order of tiny coupling parameter rendering the lifetime around $10^{26} s$ for a TeV-scale DM. Thus, it also implies the deep connection between the decaying DM with long lifetime and the active neutrinos with tiny masses.

In this paper, we consider the supersymmetric Standard Models with $R$-parity $\left(R_{p}\right)$ and three right-handed neutrinos (RHNs) $N_{i}$ where the neutrino masses are generated via the low-scale seesaw mechanism. In the DM sector, we introduce the SM singlet DM fields $\phi_{1,2}$ and a discrete $Z_{3}$ symmetry under which the term $\phi_{1} \phi_{2}$ is invariant. At the leading order they couple to leptonic sector through a dimension-seven operator

$$
\frac{1}{\Lambda} \times \frac{1}{M_{N_{l}}} \times \phi_{1} \phi_{2}\left(\frac{\mathcal{C}_{i j}^{\prime}}{M_{N_{l}}}\left(L_{i} H_{u}\right)\left(L_{j} H_{u}\right)\right),
$$


where coefficient $\mathcal{C}_{i j}^{\prime}$ come from neutrino Dirac Yukawa couplings, constrained by light neutrino masses in seesaw mechanism. It can be obtained after integrating out the heavy right-handed neutrinos (RHNs) and a superheavy field $X$ with mass $\Lambda$, provided that $\phi_{i}$ only interacts with the RHNs mediated by $X$. Note that there is a GUT scale in GUTs, we shall assume $\Lambda \sim M_{G U T}$. Interestingly, this is the exact scale that we need to produce the correct DM density via the freeze-in mechanism. In our models, the vacuum expectation values (VEVs) for $\phi_{1,2}$ are close to the RHN masses. Thus, Eq. (2) gives the small parameter $\lambda$ approximately if we identify the terms in the bracket as $m_{\nu}$. Furthermore, the preferred $\mu$ and $\tau$ decay channels can be related to the neutrino tri-bimaximal mixing (TBM) scenario with the second right-handed neutrino $N_{2}$ dominant seesaw mechanism [12]. In short, cosmic ray anomalies, if confirmed further, potentially have deep correlation with neutrino physics, especially such seesaw mechanism.

The DM relic density generally conflicts with standard thermal freeze-out scenario in the decaying DM models if the scalar components of the DM fields acquire VEVs. It is not difficult to understand it from the effective operators $\phi_{1} \phi_{2} \mathfrak{L}^{2}$ ( $\mathfrak{L}$ are the operators for particles in the MSSM) which can generate large annihilating rate. However, they also catastrophically make $\phi_{i}$ decay very fast. So one usually considered the non-thermal DM production, for example, a detail study given in Ref. [13]. Unlike the weakly interacting massive particle (WIMP) scenario, the non-thermal production usually loses the natural predication on DM abundance. Recently, it was proposed that the feebly interacting massive particle (FIMP) may be an alternative to WIMP [14], shedding light on decaying DM. Typically, the FIMP involving a coupling at the strength $\mathcal{O}\left(10^{-13}\right) \sim \mathrm{TeV} / M_{G U T}$ for the decay dominated freezein mechanism, or a larger one $\sim \mathcal{O}\left(10^{-11}\right)$ for scattering dominated freeze-in mechanism. Amazingly, in our model, $\phi_{1,2}$ must couple to the RHNs by the dimension-five operators suppressed by $M_{X}$ somewhat smaller than $M_{G U T}$ and by $M_{N_{i}} \sim 10^{4} \mathrm{GeV}$, based on proper decaying lifetime of DM. Similar results hold for the scattering process dominated freeze-in mechanism. Therefore, in our decaying DM model, its relic density again is a "miracle" via the freeze-in mechanism.

As a by product in supersymmetric SMs with freeze-in mechanism, we are able to solve the small scale problem for power spectrum, in the presence of a metastable $R_{p}$-odd state $\widetilde{\phi}$ in the DM sector. The point is the following: the whole supermultiplets $\phi_{1,2}$ are freezed into the thermal bath. We assume that $m_{\phi_{R}}+m_{\tilde{\phi}^{\prime}}>m_{\tilde{\phi}}$, where $\phi_{R}$ is the lightest state and the DM particle while $\widetilde{\phi}^{\prime}$ is the lighter $R_{p}$-odd state and has a mass close to the DM particle $\phi_{R}$. By virtue of $Z_{3} \times R_{p}$, the leading decay mode of $\widetilde{\phi}$ is to the lightest supersymmetric particle (LSP) plus $\phi_{R}$. Provided that $m_{\tilde{\phi}}$ and the LSP are respectively sufficient heavy and light, the relativistic LSP is produced from $\widetilde{\phi}$ late decay at some sufficient late time $\tau_{I} \sim 10 s-1000 s$. This warm DM component is just the key to reduce power spectrum on 
small scale [15].

In addition, we can still explain the baryon asymmetry via soft leptogenesis [16] since the seesaw scale is low around $10^{4} \mathrm{GeV}$. In supersymmetric seesaw framework, when the new CP-violating sources in the soft terms dominate the sneutrino(s) $\widetilde{N}$ CP-violating decay, the so-called soft leptogensis [17, 18] is indeed able to produce enough lepton numbers in our model. Before the discovery of gaugino effect [32], soft leptogensis suffers the highly suppressed bilinear soft mass term $B_{N_{i}} M_{N_{i}} \widetilde{N_{i}} \widetilde{N_{i}}$ with $B_{N_{i}} \lesssim 10^{-3} M_{S U S Y}$ where $M_{S U S Y}$ is the universal supersymmetry breaking scale. In this paper, we assume that the trilinear soft terms $A Y^{N i j} \widetilde{N_{i}} \widetilde{L}_{j} H_{u}$ are the only sources of CP-violation in the supersymmetry breaking soft terms. Interestingly, enough baryon number density can be generated naturally. Even the baryon number density tends to be overproduced if $M_{N_{2}}$ is too light $\sim \mathcal{O}(\mathrm{TeV})$, we can choose a relatively smaller universal $A$ term or tune its $\mathrm{CP}$ violation phase to obtain the observed baryon asymmetry.

This paper is organized as follows. In Section II, we present the model, and discuss the decay of DM, its relic density, as well as the relation between neutrino physics and cosmic ray anomalies. In Section III, we study the phenomenological consequences of our model, such as the solution to the small scale structure problem, and the low-scale soft leptogensis. In the Appendix A, we briefly review the freeze-in mechanism.

\section{THE SUPERSYMMETRIC DECAYING DARK MATTER MODEL WITH $N_{2}$ DOMINANT SEESAW MECHANISM}

\section{A. The Decaying Dark Matter Model}

We consider the supersymmetric SM with three RHNs and $R$-parity, and introduce a DM sector. As we know, a well defined DM sector should not only have a DM particle at the TeV scale, but also spontaneously breaks the discrete symmetry that stabilize the DM particle. The simplest dark sector contains a SM singlet $\phi$ and a $Z_{2}$ discrete symmetry under which only $\phi$ is odd. To have a decaying DM, we break the $Z_{2}$ symmetry by giving a VEV to $\phi$, i.e., $\langle\phi\rangle \neq 0$. Thus, at the leading order, $\phi$ couples to the observable sector through dimension-five operators $\phi^{2} N_{i}^{2} / \Lambda$, which can be obtained from a renormalizable theory after integrating out a heavy field with mass $\Lambda$. This decaying DM can explain the cosmic ray anomalies and satisfy the other phenomenological requirements that we shall consider. For an example, see Ref. [19]. However, in such an simple model it is very difficult to break $Z_{2}$ symmetry naturally. Concretely speaking, we may have to introduce another SM singlet field $S^{\prime}$ that couples to $\phi$ via a superpotential term $S^{\prime} \phi^{2}$. This superpotential term provides the quartic term to the scalar potential of $\phi$, and then we can realize the $Z_{2}$ symmetry 
breaking. After $Z_{2}$ symmetry breaking, $\phi$ and $S^{\prime}$ will mix with each other. Because we cannot forbid the direct couplings between the SM singlet $S^{\prime}$ and the observable sector, this simplest model is excluded unless we have huge fine-tuning.

Therefore, we consider a DM sector with two SM singlet fields $\phi_{1,2}$ and a discrete $Z_{3}$ symmetry. Under $Z_{3}$ symmetry, $\phi_{1,2}$ transform as follows

$$
\phi_{1} \rightarrow w \phi_{1}, \quad \phi_{2} \rightarrow w^{2} \phi_{2}
$$

where $w \equiv e^{i 2 \pi / 3}$. All the other fields in our model are neutral under the $Z_{3}$ symmetry, so, any renormalizable coupling term between the DM sector and observable sector is forbidden due to the $Z_{3}$ symmetry and $R$-parity. In particular, the traditional particle physics in the observable sector will not be affected. The most general $Z_{3}$-invariant and renormalizable superpotential in the DM sector, as well as the corresponding supersymmetry breaking soft terms are

$$
\begin{aligned}
W_{D M} & =\frac{\lambda_{1}}{3} \phi_{1}^{3}+\frac{\lambda_{2}}{3} \phi_{2}^{3}+M_{\phi} \phi_{1} \phi_{2} \\
-\mathcal{L}_{\text {soft }}^{D M} & =m_{\phi_{1}}^{2}\left|\phi_{1}\right|^{2}+m_{\phi_{2}}^{2}\left|\phi_{2}\right|^{2}+\left(\frac{A_{\lambda_{1}}}{3} \lambda_{1} \phi_{1}^{3}+\frac{A_{\lambda_{2}}}{3} \lambda_{2} \phi_{2}^{3}+B_{\phi} M_{\phi} \phi_{1} \phi_{2}+\text { h.c. }\right) .
\end{aligned}
$$

In fact, this model not only preserves $Z_{3}$-symmetry, but also a trivial $R$-parity. In the following, we shall prove that this simple DM sector can break the $Z_{3}$-symmetry spontaneously, and has a proper spectrum with a TeV-scale decaying DM coupling to the observable sector.

As poingted out in the Introduction, to have the desirable DM lifetime, abundance and decay products, the DM should couple to the RHNs via the dimension-five operators suppressed by the GUT scale $\sim M_{G U T}$. This can be achieved by integrating out a heavy SM singlet field $X$, which mediates the interactions between the DM sector and observable sector. So we consider the following superpotential

$$
\begin{aligned}
W \supset & \frac{M_{N_{i}}}{2} N_{i}^{2}+Y_{i j}^{N} L_{i} H_{u} N_{j}+\frac{\lambda_{X i}}{2} X N_{i} N_{i} \\
& +\lambda_{X \phi} \phi_{1} \phi_{2} X+\left(\frac{M_{X}}{2} X^{2}+\text { irrevelant terms }\right)
\end{aligned}
$$

with $M_{X} \sim M_{G U T}$. For simplicity, we have assumed that the RHNs are in the mass basis. We can explain the neutrino masses and mixings by employing some non-Abelian flavor symmetry such as $A_{4}$ [20], although we do not consider it here. In addition, we do not consider the superpotential $X H_{u} H_{d}$ so that we can explain the PAMELA experiment. This can be realized in the five-dimensional scenario compactified on $S^{1} / Z_{2}$ (or in the M-theory on $\left.S^{1} / Z_{2}\right)$ where $X$ and $H_{u} / H_{d}$ are localized on the different D3-branes on the two boundaries of $S^{1} / Z_{2}$ while the right-handed neutrinos are in the bulk. 
To obtain the effective action below the scale $M_{X}$, we integrate out the heavy field $X$ through its equation of motion

$$
M_{X} X+\lambda_{X \phi} \phi_{1} \phi_{2}+\lambda_{X i} N_{i}^{2}=0 .
$$

So we obtain the desirable dimension-five operators, which describe the interactions between the DM sector and RHNs. The effective superpotential are

$$
\begin{aligned}
W_{N, e f f}= & W_{h i d}-\frac{\lambda_{X \phi} \lambda_{X i}}{2 M_{X}} \phi_{1} \phi_{2} N_{i}^{2} \\
& +\left(\frac{M_{N_{i}}}{2} N_{i}^{2}+Y_{i j}^{N} L_{i} H_{u} N_{j}\right)+(\ldots),
\end{aligned}
$$

where dots denote the irrelevant corrections after integrating out $X$. Also, the corresponding supersymmetry breaking soft terms are given by

$$
\begin{aligned}
-\mathcal{L}_{\text {soft }} \supset & \mathcal{L}_{\text {soft }}^{\text {hid }}+m_{\widetilde{N}_{i}}^{2}\left|\widetilde{N}_{i}\right|^{2} \\
& +\left(\frac{\mathcal{C}^{\phi}}{2} A_{\phi N_{i}} \phi_{1} \phi_{2} N_{i}^{2}+A_{i j} Y_{i j}^{N} \widetilde{L}_{i} H_{u} \widetilde{N}_{j}+\frac{B_{N_{i}}}{2} M_{N_{i}} \widetilde{N}_{i}^{2}+\text { h.c. }\right),
\end{aligned}
$$

where

$$
\mathcal{C}^{\phi} \equiv-\frac{\lambda_{X \phi} \lambda_{X i}}{M_{X}} .
$$

Especially, the effective action described by Eqs. (7) and (8) will provide the dynamics to freeze-in the DM particles, and generate baryon asymmetry at the scale around $M_{N_{i}}$.

To obtain the effective action below the right-handed neutrino mass scale, we further integrate out the RHNs through their equations of motion

$$
M_{N_{j}} N_{j}+Y_{i j}^{N} L_{i} H_{u}-\frac{\lambda_{X \phi} \lambda_{X i}}{M_{X}} \phi_{1} \phi_{2} N_{i}=0 .
$$

Then, the leading order operators coupling $\phi_{i}$ to the SM particles are dimension-seven

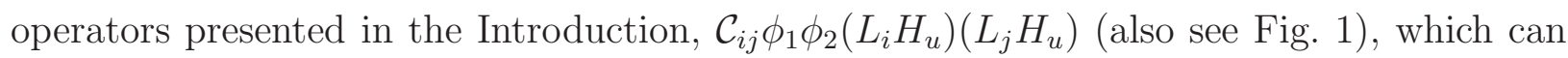
account for cosmic ray anomalies. The operator coefficients are

$$
\mathcal{C}_{i j}=-\frac{\lambda_{X \phi} \lambda_{N_{l}}}{M_{X}} \frac{Y_{i l}^{N} Y_{j l}^{N}}{M_{N_{l}}^{2}},
$$

where $l$ is summed over three RHNs. Those coefficients have a clear correlation with neutrino Dirac Yukawa couplings as well as the light neutrino Majorama mass matrix. After $Z_{3}$ symmetry breaking by the VEVs of $\phi_{i}$, we obtain the renormalizable interactions between the DM and (s)leptons from superpotential terms $\phi \nu_{i} \nu_{j}$, as well as the dimension-five interactions $\phi \nu_{i}\left(L_{j} H_{u}\right)$. The dimension-five operators are interesting since the DM particles can decay dominantly to the $\mu$ and $\tau$ leptons due to the neutrino TBM. 


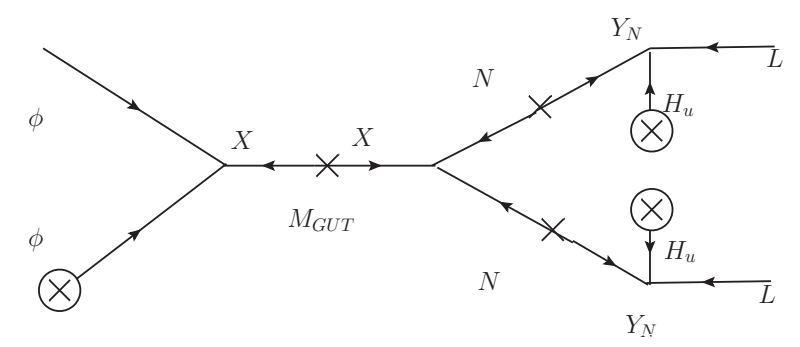

FIG. 1: Feymann diagram for the dimension-7 operators $\mathcal{C}_{i j} \phi_{1} \phi_{2}\left(L_{i} H_{u}\right)\left(L_{j} H_{u}\right) / M_{X} M_{N_{l}}^{2}$ generated by integrating out $X$ and $N_{i}$ at tree level.

\section{B. Spontaneously $Z_{3}$ Symmetry Breaking and Decaying DM}

Cosmic ray anomalies can be explained elegantly by the long-lived decaying DM with lifetime $\tau \sim 10^{26}$ s that decay dominantly to the charged leptons. Because the DM lifetime is so long, it is natural to have a symmetry if the DM is stable. In our model, this symmetry is the discrete $Z_{3}$ symmetry. To break the $Z_{3}$ symmetry spontaneously, we consider the relevant scalar potnetial $V\left(\phi_{i}\right)$ from Eq. (4)

$$
\begin{aligned}
V\left(\phi_{i}\right)= & \left|\lambda_{1} \phi_{1}^{2}+M_{\phi} \phi_{2}\right|^{2}+\left|\lambda_{2} \phi_{2}^{2}+M_{\phi} \phi_{1}\right|^{2} \\
& +m_{\phi_{1}}^{2}\left|\phi_{1}\right|^{2}+m_{\phi_{2}}^{2}\left|\phi_{2}\right|^{2}+\left(\frac{A_{\lambda_{1}}}{3} \lambda_{1} \phi_{1}^{3}+\frac{A_{\lambda_{2}}}{3} \lambda_{2} \phi_{2}^{3}+B_{\phi} M_{\phi} \phi_{1} \phi_{2}+\text { h.c. }\right) .
\end{aligned}
$$

Note that $M_{X} \gg M_{\phi_{i}}$, the contributions to the low energy effective scalar potential from the superpotential $X \phi_{1} \phi_{2}$ are very small, and then we do not consider them. Because Eq. (12) contains quite a few parameters, analytical study is pretty difficult. To reduce the parameters in the DM sector, we assume that the squared soft masses $m_{\phi_{i}}^{2}$ are universal, and the trilinear soft terms $A_{\lambda_{i}}$ are universal. Moreover, to avoid the Landau pole problem for Yukawa couplings below the GUT scale, we choose $\lambda_{1}=\lambda_{2}=0.3$.

First, we parametrize the fields $\phi_{i}$ as follows

$$
\phi_{i}=v_{i}+\frac{\phi_{i, R}^{0}+i \phi_{i, I}^{0}}{\sqrt{2}}
$$

where "0" denotes the interaction eigenstates. We require that the spectrum have the following properties: (i) The lightest scalar as the DM particle should be about 2 TeV from the Fermi-LAT electron excess at high energy region. (ii) There should be a heavy and sufficient long lived $R_{p}$-odd fermion with mass about $5 \mathrm{TeV}$ so that we can solve the small scale problem. Although these requirements impose some constraints on parameter space, they can still be satisfied easily. In the following, we present an explicit example whose 
input parameters are

$$
\begin{aligned}
& \lambda_{1}=\lambda_{2}=0.3, \quad M_{\phi}=1.0 \mathrm{TeV} \\
& m_{\phi_{i}}^{2}=200 \mathrm{GeV}^{2}, \quad B_{\phi}=-600 \mathrm{GeV}, \quad A_{\lambda_{i}}=600 \mathrm{GeV} .
\end{aligned}
$$

Let us comment on the above choice of parameters. With the fixed $\lambda_{i}$, without tuning on supersymmetry breaking soft terms, larger $M_{\phi}$ will generate larger VEVs for $\phi_{i}$ as well as heavier spectrum, which is disfavored by the DM mass requirement. Because $A_{\lambda_{i}}$ have the same sign, we choose negative $B_{\phi}$ so that the VEVs for $\phi_{i}$ have the same sign as well and we can have an absolute stable vacuum. The larger $A$-terms help us to have a more phenomenologically interesting spectrum, i.e., to increase the mass splitting between $\widetilde{\phi}$ and $\phi_{R}$ and meanwhile to keep $m_{\widetilde{\phi}}<m_{\phi_{R}}+m_{\widetilde{\phi}^{\prime}}$.

Numerically, one global minimum is located at

$$
\left\langle\phi_{1}\right\rangle \equiv v_{1} \approx-6.06 \mathrm{TeV}, \quad\left\langle\phi_{2}\right\rangle \equiv v_{2} \approx-6.57 \mathrm{TeV}
$$

At this vacuum, the various mass eigenvalues and corresponding eigenstates are respectively given by

$$
\begin{aligned}
& m_{\phi_{R}} \approx 2.60 \mathrm{TeV}, \quad m_{\phi_{R}^{\prime}} \approx 4.81 \mathrm{TeV}, \\
& m_{\phi_{I}} \approx 2.91 \mathrm{TeV}, \quad m_{\phi_{I}^{\prime}} \approx 4.83 \mathrm{TeV}, \\
& m_{\widetilde{\phi}} \approx-4.80 \mathrm{TeV}, \quad m_{\widetilde{\phi}^{\prime}} \approx-2.78 \mathrm{TeV} . \\
& \phi_{R}=0.70 \phi_{1, R}^{0}+0.71 \phi_{2, R}^{0}, \quad \phi_{R}^{\prime}=0.71 \phi_{1, R}^{0}-0.70 \phi_{2, R}^{0}, \\
& \phi_{I}=0.82 \phi_{1, I}^{0}+0.58 \phi_{2, I}^{0}, \quad \phi_{I}^{\prime}=-0.58 \phi_{1, I}^{0}+0.82 \phi_{2, I}^{0}, \\
& \widetilde{\phi}=-0.65 \widetilde{\phi}_{1}^{0}+0.76 \widetilde{\phi}_{2}^{0}, \quad \widetilde{\phi}^{\prime}=0.76 \widetilde{\phi}_{1}^{0}+0.65 \widetilde{\phi}_{2}^{0} .
\end{aligned}
$$

Thus, the lightest CP-even state $\phi_{R}$ is the DM particle accounting for the cosmic ray anomalies. $\phi_{I}, \widetilde{\phi}^{\prime}$ and $\phi_{I, R}^{\prime}$ are unstable but will contribute to the DM abundance in the freeze-in mechanism. The heavy metastable state $\widetilde{\phi}$ is crucial to solve the small scale problem on power spectrum. Notice that we have arranged parameters to have $m_{\widetilde{\phi}}<m_{\widetilde{\phi}^{\prime}}+m_{\phi_{R}}$, $\tilde{\phi}$ can not decay to $\widetilde{\phi}^{\prime}$ and $\phi_{R}$ at two-body level. We emphasize that with suitable mass $\widetilde{\phi}^{\prime}$ might also constitute a component of DM today by forbidding its two body-decay to $\phi_{R}$ and gravitino $\widetilde{G}$. By the way, all the mixing factors are nearly democratic about 0.7 , so for simplicity we may drop this factor in the following discussions, and the subscripts for $\phi_{i}$ may be ignored since they will neither affect the discussions nor bring any misunderstanding.

After the $Z_{3}$ symmetry breaking, the lightest $Z_{3}$-odd state is unstable and decays to leptons through the heavy RHNs, which is described in Fig. 1. The DM particles can decay via the operators $\mathcal{C}_{i j} \phi_{1} \phi_{2}\left(L_{i} H_{u}\right)\left(L_{j} H_{u}\right)$, and we are interested in the final sates containing $\mu$ 
and $\tau$. At the leading order, such DM decays are described by the dimension-five operators $\mathcal{C}_{i j}^{5} \phi \nu_{i}\left(L_{j} H_{u}\right)$ obtained from dimension-seven operators with one VEV for $\phi_{i}$ and one VEV for $H_{u}$. To show the close relation between DM decay and neutrino masses/TBM, we express the dimension-five operator coefficients into the light neutrino mass matrix elements. First, the Dirac neutrino mass matrix can be written as

$$
M_{L R}=Y^{N}\left\langle H_{u}^{0}\right\rangle=v \sin \beta \times\left(\mathcal{N}_{1}, \mathcal{N}_{2}, \mathcal{N}_{3}\right),
$$

where $\mathcal{N}_{i}$ is the $i$-th column, and $\tan \beta=\left\langle H_{u}^{0}\right\rangle /\left\langle H_{d}^{0}\right\rangle$ as in the MSSM, $v=$ $\sqrt{\left(\left\langle H_{u}^{0}\right\rangle\right)^{2}+\left(\left\langle H_{d}^{0}\right\rangle\right)^{2}}=174 \mathrm{GeV}$. Using the seesaw formular $M_{L L}=M_{L R} M_{R R}^{-1} M_{L R}^{T}$, we get the coefficients

$$
\begin{aligned}
\mathcal{C}_{i j}^{5} & =-\frac{\lambda_{X \phi}}{M_{X}}\left(\frac{v^{2} \sin ^{2} \beta \mathcal{N}_{l} \mathcal{N}_{l}^{T}}{M_{N_{l}}}\right)_{i j} \frac{\lambda_{X l} v_{\phi}}{v \sin \beta M_{N_{l}}} \\
& \approx-\frac{\lambda_{X \phi}}{v \sin \beta}\left(\frac{\left(M_{L L}\right)_{i j}}{M_{X}}\right)\left(\frac{v_{\phi}}{M_{N_{2}}}\right),
\end{aligned}
$$

where $v_{\phi}$ denotes $v_{1}$ or $v_{2}$, and the family universal couplings $\lambda_{X l} \simeq 1$ are assumed. This approximation is valid if $M_{N_{2}}$ dominates the seesaw contributions to $M_{L L}$ and $M_{N_{2}} \sim M_{N_{1}}$. Thus, the DM decays are closely related to the light neutrino mass matrix (elements), which will be studied in the next Section. We will show that the entries in the light neutrino mass matrix $\left(M_{L L}\right)_{i j}$ are at the same order (about the heaviest neutrino mass) for $i, j=2,3$, while the other entries are much smaller (around the second heaviest neutrino mass or smaller).

The DM branch decay lifetime is

$$
\begin{aligned}
\tau\left(\phi_{R} \rightarrow \widetilde{\nu}_{i} \ell_{j} \widetilde{H}_{u}\right) & \approx 768 \pi^{3} \times \frac{1}{\left(\mathcal{C}_{i j}^{5}\right)^{2}} \frac{1}{m_{\phi_{R}}^{3}} \\
& =3.6 \times 10^{26} \times\left(\frac{M_{X}}{10^{15} \mathrm{GeV}}\right)^{2} \times\left(\frac{0.05 \mathrm{eV}}{\left(M_{L L}\right)_{i j}}\right)^{2} \times\left(\frac{M_{N_{2}}}{10^{4} \mathrm{GeV}}\right)^{2} \\
& \times\left(\frac{5 \mathrm{TeV}}{v_{\phi}}\right)^{2} \times\left(\frac{2 \mathrm{TeV}}{m_{\phi_{R}}}\right)^{3} s
\end{aligned}
$$

where we have taken $\tan \beta=5$ throughout this paper. The actual lifetime does not depend on it much since a larger $\tan \beta$ always gives $\sin \beta \approx 1$. We keep $\lambda_{X \phi}$ as an adjustable parameter to obtain the proper lifetime of $\phi_{R}$, which will be chosen as 0.5 from then on. In order to generate the DM density via freeze-in mechanism, we choose $M_{N_{i}} / M_{X} \sim 10^{-11}$. And then we explain the neutrino masses and mixings via the low-scale seesaw mechanism. Therefore, as pointed out in the Introduction, the crucial point to get such a long lifetime decaying $\mathrm{DM}$ is the combined factor $M_{L L} / M_{X} \sim 10^{-26}$. 


\section{C. $N_{2}$ Dominant Seesaw Mechanism and Cosmic Ray Anomalies}

Although our model can generate the suitable DM lifetime naturally, the dominant decays to the leptonic final states and the fittings of the PAMELA and Fermi-LAT data need further study. Especially, the decaying product should be dominated by the second and third families of charged leptons [21]. Note that the approximate $\mu-\tau$ symmetry is introduced to explain the light neutrino masses and mixings [22], we suggest that the DM decay is related to the $N_{2}$ dominant seesaw mechanism which can explain neutrino TBM [12].

With approximate $\mu-\tau$ symmetry [22], we obtain the general light Majorana mass matrix by four parameters

$$
M_{L L}=m_{0}\left(\begin{array}{ccc}
X & Y & Y \\
Y & Z & W \\
Y & W & Z
\end{array}\right) .
$$

It predicts the maximal atmosphere mixing angle $\theta_{23}=\pi / 4$ and $\theta_{13}=0$, but leave the solar mixing angle $\theta_{12}$ arbitrary. Taking $\sin ^{2} 2 \theta_{12}=8 / 9$, the neutrino TBM is obtained [23]. The TBM $M_{L L}$ mass matrix only has three parameters since this fixed $\theta_{12}$ is equivalent to a relation $Z+W=X+Y$. So we have

$$
M_{L L}=m_{0}\left(\begin{array}{ccc}
X & Y & Y \\
Y & X+V & Y-V \\
Y & Y-V & X+V
\end{array}\right) .
$$

In the framework of seesaw mechanism with heavy RHN dominance, the crucial point of neutrino mixings is the specially aligned Dirac neutrino mass matrix (or say the Yukawa coupling matrix). Concretely speaking, the neutrino TBM can be understood by the aligned vacuum from an $A_{4}$ discrete flavour symmetry breaking [24].

To explain why the DM decays dominant to muon and tau via neutrino physics, we modify the original Dirac Yukawa coupling ansatz used in Ref. [12] as follows

$$
M_{L R}=\left(\begin{array}{ccc}
A & 0 & 0 \\
A & -B & 0 \\
A & B & C
\end{array}\right) .
$$

To produce the realistic neutrino masses and mixings, we assume three RHNs with proper mass hierarchy $M_{N_{1}} \lesssim M_{N_{2}} \ll M_{N_{3}}$ so that the light neutrino mass matrix accommodates both the TBM and the $\mu+\tau$ dominated decay product of DM. Thus, the light neutrino 
mass matrix is

$$
\begin{aligned}
M_{L L} & =v^{2} \sin ^{2} \beta\left(\frac{\mathcal{N}_{1} \mathcal{N}_{1}^{T}}{M_{N_{1}}}+\frac{\mathcal{N}_{2} \mathcal{N}_{2}^{T}}{M_{N_{2}}}+\frac{\mathcal{N}_{3} \mathcal{N}_{3}^{T}}{M_{N_{3}}}\right) \\
& =m_{0}\left(\begin{array}{ccc}
X & Y & Y \\
Y & X+V & Y-V \\
Y & Y-V & X+V
\end{array}\right)+\mathcal{O}\left(C^{2} / M_{N_{3}}\right)
\end{aligned}
$$

where $X=Y=A^{2} /\left(M_{N_{1}} m_{0}\right)$, and $V=B^{2} /\left(M_{N_{2}} m_{0}\right)$. The last term gives the subdominant contributions to $M_{L L}$, but it is still important for the mass of the lightest neutrino.

Now we show that the DM dominant decay channel to $\mu+\tau$ is a natural result for the $N_{2}$ dominant seesaw mechanism if the neutrino masses are normal hierarchy. Combining the DM decays with neutrino masses and TBM gives some contraints on the free parameters. First, it is obvious that $M_{L L}$ should be in the second RHN dominance. Next, with Eq. (24) we obtain three neutrino approximate masses

$$
m_{\nu_{3}} \approx 2 V m_{0}=\frac{2 B^{2}}{M_{N_{2}}}, \quad m_{\nu_{2}} \approx 3 X m_{0}=\frac{3 A^{2}}{M_{N_{1}}}, \quad m_{\nu_{1}} \lesssim \mathcal{O}\left(\frac{C^{2}}{M_{N_{3}}}\right),
$$

in a normal hierarchy form. Thus, the neutrino oscillation data $\Delta m_{21}^{2} \approx 7.65 \times 10^{-5} \mathrm{eV}^{2}$ and $\Delta m_{31}^{2} \approx 2.40 \times 10^{-3} \mathrm{eV}^{2}$ suggest that

$$
\frac{B^{2}}{M_{N_{2}}}: \frac{A^{2}}{M_{N_{1}}} \simeq 8.4: 1
$$

is valid when the $N_{3}$ is sufficient heavy [25]. But from Eq. (19), the dimension-five operator coefficients are proportional to $1 / M_{N_{l}}^{2}$. And then they disfavor large hierarchy $M_{N_{2}} \gg M_{N_{1}}$. So the hierarchy in Eq. (26) is mainly due to the moderate relation $A<B$. Because $B^{2} / M_{N_{2}}$ will appear several times later, we fix it in the massless $\nu_{1}$ limit (or say infinite $m_{N_{3}}$ limit)

$$
\frac{B^{2}}{M_{N_{2}}} \approx \sqrt{\Delta m_{31}^{2} / 2} \approx 0.035 \mathrm{eV} .
$$

The DM $\phi_{R}$ decays to the SM fermions are the dominant primary source of comic ray since the lifetime of other states such as $\widetilde{\phi}$ is much short at the cosmic time scale. At tree level, the $\phi_{R}$ three-body decay modes are

$$
\phi_{R} \rightarrow \ell_{i} H_{u} \nu_{j}, \quad \widetilde{\ell}_{i} \widetilde{H}_{u} \nu_{j}
$$

and the corresponding lifetime estimation is given in Eq. (20). In Ref. [10], it has been explicitly simulated the electron spectra, and found that the spectra from direct hard leptons plus the soft contributions from cascade decays via the slpeptons and Higgs, Higgsinos are able to fit the PAMELA and Fermi-LAT experiments while not produce the anti-proton 
excesses in the constrained MSSM. Moreover, for the two-body decay modes, $\phi_{R}$ decays to pure (two) neutrinos. The branch decay lifetime is approximately given by

$$
\tau\left(\phi_{R} \rightarrow \nu_{i} \nu_{j}\right) \approx 8 \pi \times \frac{1}{\left(\mathcal{C}_{i j}^{5}\right)^{2}} \frac{1}{m_{\phi_{R}}^{3}}\left(\frac{m_{\phi_{R}}}{v \sin \beta}\right)^{2},
$$

which is about $20 \%$ of the one through three-body decays. Assuming a lifetime about $5 \times 10^{26}$ s for three-body decay modes to explain the comic ray anomalies, we have $\tau\left(\phi_{R} \rightarrow\right.$ $\left.\nu_{i} \nu_{j}\right) \sim 10^{26}$ s. The produced neutrino signals are potentially detectable with the upcoming IceCube neutrino observatory [26], and the constraints on the DM models can be found in Ref. [27].

\section{DM Density from Freeze-in Mechanism}

The decaying DM abundance can be produced naturally via the freeze-in mechanism (for a brief review, see Appendix (A) in our models. Let us explain the cosmological setup first since it is important to make freeze-in mechanism work. The initial relic density of $\phi_{i}$ should almost vanish, while (s)RHNs have the thermal density at least at $T \sim M_{N}$. However, for the low-scale seesaw mechanism in the MSSM, the (s)RHNs only weakly interact with the plasma because the neutrino Dirac Yukawa couplings are small about $10^{-5}$. Thus, after inflation the MSSM particles in the plasma alone cannot produce the thermal (s)RHNs. But this problem can be solved easily in the Next to the MSSM (NMSSM), where the superpotential term $S N_{i}^{2}$ can be introduced. Also, the suitable density for the (s)RHNs can be produced non-thermally by coupling them directly to the inflaton field. Thus, we assume the (s)RHNs in the plasma at the temperature $T \gtrsim M_{N_{i}}$. But the initial densities for $\phi_{i}$ are ignorable since they are SM singlets and only very weakly interacts with the (s)RHNs.

However, during the decoupling of $\widetilde{N}_{i}$, in the absence of inverse decay, the tiny branch decays or the scattering processes of $\widetilde{N}_{i}$ and $N_{i}$ produces $\phi_{i}$. To have the natural relic densities of $\phi_{i}$ via freeze-in mechanism, the typical couplings are required to be around $10^{-13}$ for two-body decays and $10^{-11}$ for two to two scattering processes [14]. To be concrete, we give the relevant terms between $(\mathrm{s}) \mathrm{RHNs}$ and $\phi_{i} / \widetilde{\phi}_{i}$ for freeze-in mechanism

$$
\begin{gathered}
-\mathcal{L} \supset \mathcal{C}^{\phi}\left(\phi_{1} \widetilde{\phi}_{2}+\phi_{2} \widetilde{\phi}_{1}\right) \widetilde{N}_{i} N_{i}+\frac{\mathcal{C}^{\phi}}{2} \widetilde{\phi}_{1} \widetilde{\phi}_{2} \widetilde{N}_{i}^{2}+\left|F_{\phi_{1}}\right|^{2}+\left|F_{\phi_{2}}\right|^{2}+\left|F_{N_{i}}\right|^{2} \\
\rightarrow \mathcal{C}^{\phi}\left(v_{1} \widetilde{\phi}_{2}+v_{2} \widetilde{\phi}_{1}\right) \widetilde{N}_{i} N_{i}+\mathcal{C}^{\phi} M_{N_{i}}\left(\phi_{1} \phi_{2} \widetilde{N}_{i} \widetilde{N}_{i}^{*}+\text { h.c. }\right)+(\ldots)
\end{gathered}
$$

where we only consider the dominant terms, and dots denote many ignored terms, such as the supersymmetry breaking trilinear soft terms since the freeze-in amplitudes are controlled by $M_{N_{i}} \gg A_{i j}$. Similarly, the scattering processes are also sub-dominated sinces they are 
proportional to $v_{i}$ which are several times smaller than $M_{N_{i}}$ in our model. In the precise calculations, one has to transform the interaction eigenstates to the mass eigenstates. For the DM state transformations, please see Eq. (17). In the following analysis we shall show that the DM relic density can indeed be obtained through the freeze-in mechanism, and the order-one mixing factor is not considered for simplicity. The mass eigenstates for $\widetilde{N}_{i}$ are

$$
\widetilde{N}_{i, \pm}=\frac{1}{\sqrt{2}}\left(\widetilde{N}_{i} \pm \widetilde{N}_{i}^{*}\right)
$$

where the squared mass eigenvalues are respectively given by $M_{\widetilde{N}_{i, \pm}}^{2}=M_{N_{i}}^{2}+m_{\widetilde{N}_{i}}^{2} \pm B_{N_{i}}$. Here, $m_{\widetilde{N}_{i}}^{2}$ are the soft mass square for the sRHNs.

First, the freeze-in FIMPs from $\widetilde{N}_{i,+}$ and $N_{i}$ two-body decays $\widetilde{N}_{i,+} \rightarrow N_{i} \widetilde{\phi}$ and $N_{i} \rightarrow \widetilde{N}_{i,-} \widetilde{\phi}$ as well as $\widetilde{N}_{i,+} \rightarrow \widetilde{N}_{i,-} \phi_{I R}$ are in general kinetically forbidden. In fact, in the natural soft mass scale around $\mathcal{O}(1 \mathrm{TeV})$, the mass splittings among $\widetilde{N}_{i,+}, \widetilde{N}_{i,-}$ and $N_{i}$ are about $M_{S U S Y}^{3} / M_{N_{i}}^{2}$, which at most are tens of GeVs. Consequently there are no decay channels. In fact, it is required for proper DM relic density since the typical couplings given above are $\sim M_{N_{i}} / M_{X} \gg 10^{-13}$. In short, these two-body decays must be forbidden (or at least suppressed sufficiently), otherwise, the freeze-in mechanism tends to over freeze $\mathrm{DM}(\mathrm{s})$ into the plasma. We have to point out that the above conclusion holds only when there are no mixings among the RHNs. If there exist the mixings $\epsilon_{i j}$ in a complete model, we have to require that $\epsilon_{i j} \operatorname{Max}\left\{M_{N_{i}}, M_{N_{j}}\right\} / M_{X} \sim 10^{-13}$ since the mass splittings between the RHNs should be small enough to suppress the transition between $N_{i}$ and $N_{j}$ significantly.

The scattering process $\widetilde{N}_{i} \widetilde{N}_{i} \rightarrow \phi_{I R} \phi_{I R}$ from the second line of Eq. (30) can produce the phenomenologically important components $\phi_{I R}$. The scattering process $\widetilde{N}_{i} N_{i} \rightarrow \widetilde{\phi} \phi_{I R}$ can be studied similarly, so we will not present it in this paper. Numerically, the exact coincidence is a result of the dimension-five operators $\phi_{1} \phi_{2} N_{i} N_{j} / M_{X}$. Note that the RHNs have masses about $10 \mathrm{TeV}$, and $M_{X}$ can be chosen a little bit smaller than $M_{G U T}$, we obtain $M_{N_{i}} / M_{X} \sim 10^{-11}$ at the desired order. For the scattering processes, we consider the interaction eigenstates as the mass eigenstates for simplicity since the mixings are democratic. The total cross sections are simply given by

$$
\sigma\left(\widetilde{N}_{i} \widetilde{N}_{i} \rightarrow \phi \phi\right)=\frac{\lambda_{N_{i}}^{2}}{8 \pi s} \lambda^{-1 / 2}\left(1, x_{\widetilde{N}_{i}}, x_{\widetilde{N}_{i}}\right) .
$$

The function from phase space $\lambda(a, b, c) \equiv(a-b-c)^{2}-4 b c\left(x_{I} \equiv m_{I}^{2} / s\right)$ implies that only the lighter species (at least lighter than $\widetilde{N}_{i}$ ) could be freezed into plasma with significant number densities. Numerically, the integral factor $\mathcal{I}[x, z]$ in Eq. (A7) is about 0.5 , so the 
relic densities of $\phi_{I R}$ and $\widetilde{\phi}$ are estimated to be at the right order

$$
\begin{aligned}
\Omega_{\phi} h^{2} & \sim \frac{6.0 \times 10^{22} g_{\widetilde{N}_{i}}^{2}}{g_{*}^{S} \sqrt{g_{*}^{\rho}}}\left(\frac{m_{\phi}}{M_{N_{i}}}\right) \lambda_{N_{i}}^{2} \\
& =0.065\left(\frac{m_{\phi}}{2.5 \mathrm{TeV}}\right)\left(\frac{10 \mathrm{TeV}}{M_{N_{i}}}\right)\left(\frac{229^{3 / 2}}{g_{* M S S M}}\right)\left(\frac{\lambda_{N_{i}}}{5 \times 10^{-11}}\right)^{2},
\end{aligned}
$$

where $m_{\phi}$ denotes the mass of $\phi_{I R}$ or $\widetilde{\phi}$. The relic density of gravitino $\widetilde{G}$, which comes from the non-thermal production via the $\widetilde{\phi}$ late decay, is about one order smaller than $\Omega_{\phi_{R}}$ due to the mass ratio $m_{\phi_{R}} / m_{\widetilde{G}} \sim 10$. We emphasize that this is not the final DM relic density, and the actual DM density is obtained by calculating all the processes with exact mixing factors. However, our results are enough to show that we can generate the correct DM relic density in our parameter space.

\section{PHENOMENOLOGICAL CONSEQUENCES}

\section{A. Warmed $\widetilde{G}$ and Small Scale Problem}

In our model, both $\phi_{R}$ and $\widetilde{\phi}$ are generated with equal number densities via the freezein mechanism. Because $\widetilde{\phi}$ is a relatively heavy metastable $R_{p}$-odd state, it will decay and produce some relativistic particles. Thus, we can solve the small scale problem on power spectrum if the relativistic particle is the DM candidate like the LSP in the MSSM. If the comoving free-streaming scales of the relativistic particles, i.e., their motion in the comoving framework from their production time $t_{I}$ till to the matter and radiation equality era $t_{E Q} \approx 2.2 \times 10^{12} \mathrm{~s}$, can reach the small scale $\mathcal{O}(0.1) \mathrm{Mpc}$, the power spectrum on small scale can be reduced [28]. Such warm DM scenario was proposed in Ref. [15].

To solve the small scale problem in our model, we require that $\widetilde{\phi}$ have a proper mass (about $5 \mathrm{TeV}$ in our example parameters) and a proper lifetime. Lifetime is fine in our model. Since $\widetilde{\phi}$ is odd under $Z_{3} \times R_{p}$, its leading decay mode is given by (as mentioned previously, in our interesting parameter space $\widetilde{\phi}$ can not decay to $\widetilde{\phi}^{\prime}$ and $\phi_{R}$ at two-body level)

$$
\widetilde{\phi} \rightarrow \widetilde{G}+\phi_{R} \rightarrow \ldots
$$

Because the decay rate is suppressed by $1 / M_{\mathrm{Pl}}^{2}$ in gravity mediation, the $\widetilde{\phi}$ lifetime can be sufficiently long about 10 - 1000 s, and can be even longer depending on mass splitting between the bosonic and fermionic states. However, if $\widetilde{G}$ is not the LSP, the above decay chain will produce a LSP such as neutralino. Thus, we have two viable solutions: (i) $\widetilde{G}$ itself is the LSP with mass about $\mathcal{O}(100) \mathrm{GeV}$, then it is warmed enough to reduce power 
spectrum on small scale; (B) $\widetilde{G}$ is not the LSP and has mass $\gtrsim \mathcal{O}(\mathrm{TeV})$. We require that $\widetilde{G}$ can produce the warm LSP via its decay while not forbid the two-body decay of $\widetilde{\phi}$. In short, these viable solutions do not conflicts with the parameter space in the MSSM.

Because the late decay of $\widetilde{G}$ may spoil the successful predication of big bang primary nucleosynthesis (BBN), we consider $\widetilde{G}$ as the LSP for simplicity. In fact, this process can be regarded as a method of non-thermal production of $\widetilde{G}$. The comoving free-streaming scale of a freely propagating particle can be calculated from the formular [15]

$$
\begin{aligned}
R_{f} & =\int_{t_{I}}^{t_{E Q}} \frac{v\left(t^{\prime}\right)}{a\left(t^{\prime}\right)} d t^{\prime} \\
& \simeq 2 v_{0} t_{E Q}\left(1+z_{E Q}\right)^{2} \log \left(\sqrt{1+\frac{1}{v_{0}^{2}\left(1+z_{E Q}\right)^{2}}}+\frac{1}{v_{0}^{2}\left(1+z_{E Q}\right)}\right),
\end{aligned}
$$

where $z_{E Q}$ and $t_{E Q}$ are the red shift and comic time at the matter-radiation equality era. Also, $v_{0}$ is the current velocity of $\widetilde{G}$

$$
v_{0}=\frac{T_{0}}{T_{I}} \frac{E_{I}}{m_{\widetilde{G}}},
$$

where $T_{0} \approx 2.73 \mathrm{~K}$, and $E_{I}$ and $T_{I}$ are respectively the energy and temperature when warm $\widetilde{G}$ is produced. According to Eq. (35), in order to explain the small-scale structure, $v_{0}$ should take the value $10^{-8}-10^{-7}[15]$.

If $\widetilde{G}$ is light, $v_{0}$ is not dependent on its mass $m_{\widetilde{G}}$. Thus, with the proper mass for $\widetilde{\phi}$ in our example, we can indeed solve the small scale problem. Let us explain it in details. The two-body decay rate of $\tilde{\phi}$ to its partner $\phi_{R}$ plus gravitino is calculated to be [29]

$$
\Gamma_{I}=\frac{1}{48 \pi} \frac{m_{\widetilde{\phi}}^{5}}{m_{\widetilde{G}}^{2} M_{\mathrm{P}}^{2}}\left\{1-\left(\frac{m_{\phi_{R}}}{m_{\tilde{\phi}}}\right)^{2}\right\}^{4},
$$

with the reduced Planck mass $M_{\mathrm{P}} \equiv M_{\mathrm{Pl}} / \sqrt{8 \pi} \simeq 2.4 \times 10^{18} \mathrm{GeV}$. Notice that this result is only valid when gravitino is much lighter than the mass splitting between particle and its superpartner, this is the exact situation needed in our model: $\widetilde{G}$ has a large velocity (warm enough) when it was produced. According to Eq. (37), the cosmological temperature (in the radiative dominant era) is given by $T_{I}=\left(0.301 g_{*}^{-1 / 2} M_{\mathrm{Pl}} / t_{I}\right)^{1 / 2}$, where $g_{*}$ is the effective relativistic degree of freedom in the plasma, and $t_{I}=1 / \Gamma_{I} \sim \mathcal{O}(10) s$ with $m_{\widetilde{G}} \sim 100 \mathrm{GeV}$. Then we can parametrize $v_{0}$ as follows

$$
v_{0}=1.8 \times 10^{-8} \times\left(\frac{g_{*}}{3.36}\right)^{1 / 4}\left(\frac{m_{\widetilde{\phi}}}{5 \mathrm{TeV}}\right)^{3 / 2}\left(\frac{\Delta m_{\phi}^{2}}{20 \mathrm{TeV}^{2}}\right),
$$

where $\Delta m_{\phi}^{2} \equiv m_{\tilde{\phi}}^{2}-m_{\phi_{R}}^{2}$, and we have used $E_{I}=m_{\tilde{\phi}} / 2\left(1-m_{\phi_{R}}^{2} / m_{\widetilde{\phi}}^{2}\right) \approx m_{\tilde{\phi}} / 2$. As pointed out at the beginning, $v_{0}$ does not depend on $m_{\widetilde{G}}$ explicitly, and the solution to the small-scale problem only depends on the mass of $\widetilde{\phi}$. 


\section{B. Baryon Asymmetry via Soft Leptogenesis}

Interestingly, we can also explain baryon asymmetry via the soft leptogensis, since the proper decay rate of $\phi_{R}$ requires a low-scale seesaw mechanism with $M_{N_{i}} \sim 10^{4} \mathrm{GeV}$ at least for $i=1,2$. Consequently, the new lepton number violation and $\mathrm{CP}$ violation in the supersymmetry breaking soft terms play major role in soft leptogensis. The soft leptogensis can work only well below $M_{N_{i}}<10^{9}$ [17, 18], which is the low bound on the right-handed neutrino mass in thermal leptogensis. And then reheating temperature is well below $10^{9}$ $\mathrm{GeV}$ as well. Thus, we can reduce the gravitino density produced by thermal scatterings in the thermal bath, and then the $\widetilde{G}$ late decay will not destroy BBN [30]. In short, the possible gravitino problem in the thermal leptogenesis can be solved. For a complete review, please see Ref. [31]. In our model, the relevant Lagrangian for soft leptogenesis is

$$
\begin{aligned}
-\mathcal{L} \supset & \frac{1}{\sqrt{2}} \widetilde{N}_{2+}\left(Y_{i 2}^{N}\right)^{*}\left(M_{N_{2}}+A_{i 2}^{*}\right) \widetilde{L}_{i}^{\dagger} H_{u}^{\dagger}+\frac{1}{\sqrt{2}} \widetilde{N}_{2-}\left(Y_{i 2}^{N}\right)^{*}\left(M_{N_{2}}-A_{i 2}^{*}\right) \widetilde{L}_{i}^{\dagger} H_{u}^{\dagger} \\
& +\left(Y_{i 2}^{N}\right)^{*}\left(\widetilde{N}_{2+}-\widetilde{N}_{2-}\right) L_{i}^{\dagger} \widetilde{H}_{u}^{\dagger}+\frac{1}{2} M_{2} \widetilde{\lambda}_{2} \widetilde{\lambda}_{2}+\text { h.c. },
\end{aligned}
$$

where $A_{i 2}=\left|A_{i 2}\right| e^{i \theta_{A_{i 2}}}$, and all the other soft terms have been taken real. Moreover, the $S U(2)_{L}$ gaugino mass $M_{2}$ is assumed to be real so that it will not induce large CP violation in the MSSM.

In our model, the dominant contributions to the lepton number production come from the interference between the tree-level decays of $\widetilde{N}_{i}$ and the vertex corrections with gaugino running in the loop, which are given in Fig. 2, The original soft leptogensis relies on the self-energy corrections to $\widetilde{N}_{i}$, and it is the small mass splitting (controlled by the bilinear soft terms $B_{N_{i}} M_{N_{i}} \widetilde{N}_{i}^{2}$ ) between the two real degrees of freedom of $\widetilde{N}_{i}$ denoted with $\widetilde{N}_{i \pm}$ that resonantly enhances their CP-violation decays, see Fig. 2. To make the resonant effect large enough, $B_{N_{i}} \lesssim 10^{-3} M_{S U S Y}$ must be fine-tuned to be very small [17, 18]. Later, it was found that the vertex corrections to $\widetilde{N}_{i}$ decays with gaugino running in the loop contribute to the lepton number asymmetry in a very different way, and then the normal value of $B_{N_{i}}$ is allowed [32]. This is important for our model to have successful soft leptogensis because its UV completion does not suppress $B_{N_{i}}$.

In the previous Section, we have considered the $N_{2}$ dominant seesaw mechanism to produce $M_{L L}$. Corresponding to it, this dominance again dominantly generate the lepton asymmetry. This can be seen clearly from the explicit calculations of the lepton asymmetry 
produced by a single $\widetilde{N}_{i}$ decays to lepton flavor $\alpha$, using the procedure provided in Ref. [31]

$$
\begin{aligned}
\epsilon_{i, \alpha} & \equiv \frac{\gamma\left(\widetilde{L}_{\alpha} H_{u}\right)+\gamma\left(L_{\alpha} \widetilde{H}_{u}\right)-\gamma\left(\widetilde{L}_{\alpha}^{\dagger} H_{u}^{\dagger}\right)-\gamma\left(L_{\alpha}^{\dagger} \widetilde{H}_{u}^{\dagger}\right)}{\sum_{\beta} \gamma\left(\widetilde{L}_{\beta} H_{u}\right)+\gamma\left(L_{\beta} \widetilde{H}_{u}\right)+\gamma\left(\widetilde{L}_{\beta}^{\dagger} H_{u}^{\dagger}\right)+\gamma\left(L_{\beta}^{\dagger} \widetilde{H}_{u}^{\dagger}\right)} \\
& \approx \frac{3 \alpha_{2}\left|Y_{\alpha i}^{N}\right|^{2}}{4 \sum_{\beta}\left|Y_{\beta i}^{N}\right|^{2}} \frac{M_{2}}{M_{N_{i}}} \log \frac{M_{2}^{2}}{M_{2}^{2}+M_{N_{i}}^{2}}\left(-\frac{\left|A_{i 2}\right|}{M_{N_{i}}} \sin \theta_{A_{i 2}}\right) \Delta_{B F}(T),
\end{aligned}
$$

where $\alpha_{2}=g_{2}^{2} / 4 \pi$ and $g_{2}$ is $S U(2)_{L}$ gauge coupling. This shows the $N_{2}$ dominant contributions from Eqs. (18) and (26), while the others are sub-dominant. Note that $Y^{N_{i}} \sim 10^{-5}$ in our model, the contributions from self-energy corrections suppressed by $\left|Y^{N_{i}}\right|^{2} / \alpha_{2}$ are completely ignorable. $\Delta_{B F}(T)$, whose expression is given in Ref. [18], denotes for the thermal effect in the thermal average of decay rates $\gamma$. Without it the above asymmetry vanishes at zero temperature field theory due to the exact cancellations between the fermionic and bosonic decay channels of $\widetilde{N}_{i}$. By the way, our results are consistent with a previous work in Ref. [33] which used a different calculation method.
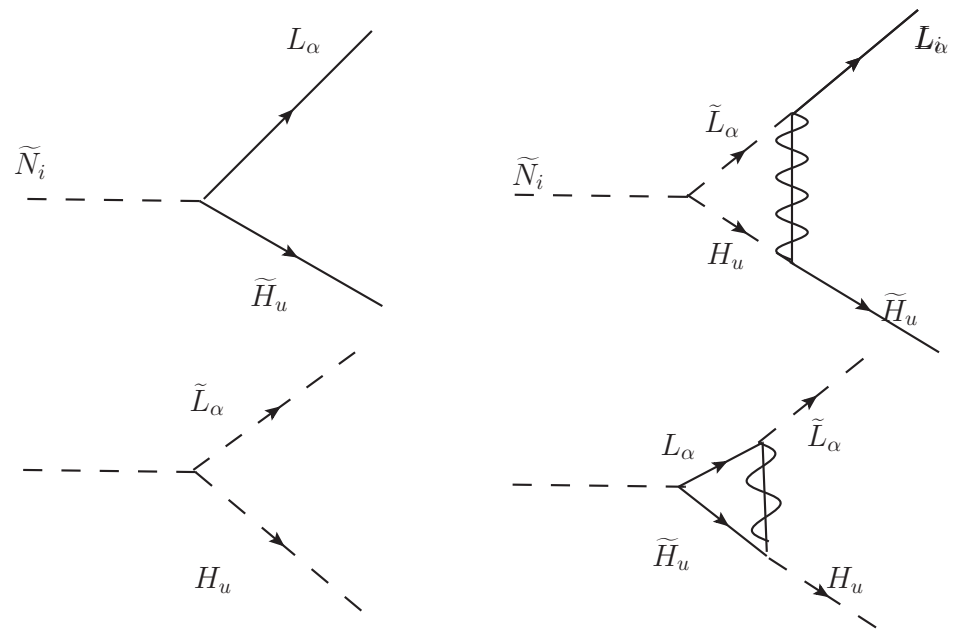

FIG. 2: Lepton number and CP-violation decays of $\widetilde{N}_{i}$ with gaugino running in the vertex correction loop. Self-energy contributions are ignored since they are suppressed by the extra Yukawa couplings $Y^{N_{i}}$.

The evolution of $\widetilde{N}_{i}$ lepton number violation decay and the evolution of $\alpha(\alpha=e, \mu, \tau)$ lepton flavor number are described by Boltzmann equations (BEs). Since the pure MSSM interaction conserves the charge $\Delta_{\alpha} \equiv\left(B_{f}+B_{s}\right) / 3-\left(L_{f}+L_{s}\right)_{\alpha}$, where $\left(L_{f}\right)_{\alpha}=\ell_{\alpha}+e_{\alpha}$ and $L_{s}$ is the total lepton number in scalar leptons. Similar definition applies to the $B_{f, s}$. It is 
convenient to study the evolution of density $\Delta_{\alpha}$, and the coupled BEs are [36]

$$
\begin{aligned}
\Delta_{\widetilde{N}}^{\prime} & =-\sum_{\alpha} S_{\alpha}(z)-\left(Y_{\widetilde{N}}^{e q}\right)^{\prime}, \quad S_{\alpha}(z)=\frac{z}{Y_{\widetilde{N}}^{e q}} \frac{\gamma_{\alpha}(z)}{s H_{N}} \Delta_{\widetilde{N}} \\
Y_{\Delta_{\alpha}}^{\prime} & =-\epsilon_{\alpha}(z) S_{\alpha}(z)+W_{\alpha}(z) \sum_{\beta}\left(A_{\alpha \beta}+C_{\beta}\right) Y_{\Delta_{\beta}},
\end{aligned}
$$

where the derivative is on $z \equiv M_{\widetilde{N}} / T$, and $\Delta_{\widetilde{N}} \equiv Y_{\widetilde{N}}-Y_{\widetilde{N}}^{e q}$. In this crude set of BEs, we consider the $\Delta L=1$ two to two scattering processes that provide the CP asymmetry source, as well as the wash-out from the top quark and gauge boson interactions. Also, the flavor effect is kept for the low-scale soft leptogensis via the $A$ matrix which expresses $Y_{\ell_{\alpha}}$ as the linear combination of $Y_{\Delta_{\alpha}}$ [34], and via the $C$ matrix which relates $Y_{H_{u}}$ to $Y_{\Delta_{\alpha}}$ [35]

$$
A=\frac{1}{207} \times\left(\begin{array}{ccc}
-64 & 5 & 5 \\
5 & -64 & 5 \\
5 & 5 & -64
\end{array}\right), \quad C_{\beta}=\frac{1}{9} \sum_{\alpha} A_{\alpha \beta}=-\frac{2}{69}\left(\begin{array}{l}
1 \\
1 \\
1
\end{array}\right)
$$

Our matrix is different from that in Refs. [33, 36] since in this model the soft leptogenesis proceeds during the era $T \sim M_{N} \simeq 10^{4} \mathrm{GeV}$, where all the Yukawa couplings and the CKM mixings are in the chemical equilibrium. Also, the $C$ matrix is included here since its entries are larger than the mixing entries in $A$ matrix. Moreover, in the simplified BEs, the source term and wash-out terms can be rewritten analytically as follows

$$
S_{\alpha}(z)=z \frac{K_{1}(z)}{K_{2}(z)} \mathcal{K}_{\alpha}, \quad W_{\alpha}(z)=\frac{1}{4} z^{3} K_{1}(z) \mathcal{K}_{\alpha},
$$

where the object $\mathcal{K}_{\alpha}$ describes the degree of washout for a single flavor $\alpha$

$$
\begin{aligned}
\mathcal{K}_{\alpha} & \equiv \frac{\Gamma_{\alpha}+\widetilde{\Gamma}_{\alpha}}{H_{N}}=\frac{m_{\alpha}}{m_{M S S M}^{*}}, \\
m_{\alpha} & =\left|Y_{\alpha 2}^{N}\right|^{2} v^{2} \sin ^{2} \beta / M_{N_{2}}
\end{aligned}
$$

where $m_{\alpha}$ is equal to $|B|^{2} / M_{N_{2}}$ for $\alpha=2,3$ while vanishes for $\alpha=1$. In the MSSM using $g_{*}=228.75$ at $T \sim M_{N}$, one obtains $m_{M S S M}^{*} \approx \sin ^{2} \beta \times 1.58 \times 10^{-3} \mathrm{eV}$. Thus, $\mathcal{K}_{2,3} \sim 20$, and then the soft leptogensis is in the strong wash out region [31, 34]. Finally, the sphaleron processes transform the survival lepton asymmetry into baryon asymmetry, eventually gives the baryon number density

$$
Y_{B}^{M S S M}=\frac{n_{B}-n_{\bar{B}}}{s}=\frac{10}{31} \sum_{\alpha} Y_{\Delta_{\alpha}} .
$$

With the initial density of $\widetilde{N}_{i}$ in thermal equilibrium required by a successful freeze-in mechanism, we present the numerical solutions to the baryon asymmetry evolution in Fig. 3 , 
The observed baryon asymmetry $Y_{B}=(8.75 \pm 0.23) \times 10^{-11}$ [37] is generated with the following parameters: $M_{N_{2}}=10^{4} \mathrm{GeV}, M_{2}=250 \mathrm{GeV}$ and $\left|A_{N_{22}}\right|=300 \mathrm{GeV}$ with phase $\theta_{A_{22}}=-1 / 4$. As the RHN mass decreases, for instance, $M_{N_{2}}=4 \times 10^{3} \mathrm{GeV}$, the baryon asymmetry tends to be overproduced. The reason is that the lepton asymmetry given in Eq. (40) is proportional to $1 / M_{N_{2}}^{2}$ but linear to $A_{N_{2}}$ and $M_{2}$. Because we have assumed that the LSP $\widetilde{G}$ has mass about $200 \mathrm{GeV}, M_{2}$ can not be too small. Therefore, we can choose a smaller $\left|A_{N_{22}}\right|=100 \mathrm{GeV}$ with phase $\theta_{A_{22}}=-1 / 5$, or we can fine-tune the phase of $A_{N_{22}}$. Anyway, the observed baryon asymmetry can be obtained in the general parameter space.
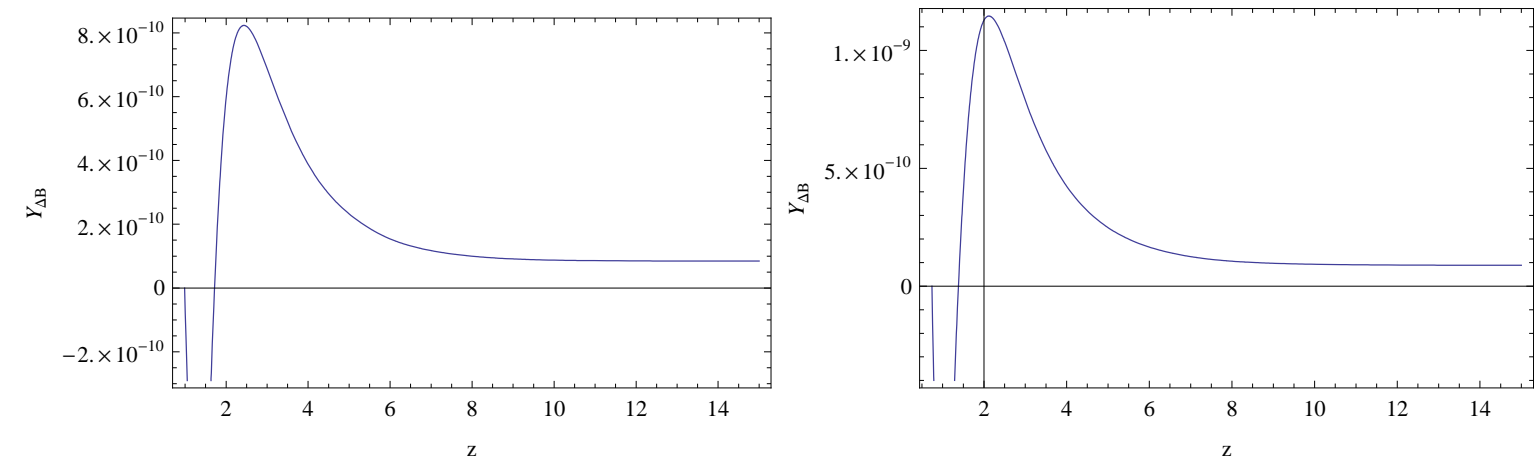

FIG. 3: Baryon asymmetry $Y_{\Delta B}(z)$ versus $z=M_{N_{2}} / T$. Left: $M_{N_{2}}=10^{4} \mathrm{GeV}, M_{2}=250 \mathrm{GeV}$ and $\left|A_{N_{22}}\right|=300 \mathrm{GeV}$ with $\theta_{A_{22}}=-1 / 4$. Right: $M_{N_{2}}=4 \times 10^{3} \mathrm{GeV}, M_{2}=250 \mathrm{GeV},\left|A_{N_{22}}\right|=100$ $\mathrm{GeV}, \theta_{A_{22}}=-1 / 5$. Initial density of $\tilde{N}_{2}$ is taken as thermal density.

\section{DISCUSSIONS AND CONCLUSION}

Cosmic ray anomalies from the Fermi-LAT and PAMELA experiments can be naturally explained by the TeV-scale decaying $\mathrm{DM}$ with a very long lifetime $\sim 10^{26}$ s which decays dominantly to the muon and tau leptons. Note that the neutrino TBM can be realized elegantly via the $\mu-\tau$ symmetry, we conjectured that the DM decay is related to the neutrino physics. We considered the supersymmetric Standard Model with three righthanded neutrinos. To realize the decaying DM, we introduced a $Z_{3}$ discrete symmetry and two DM particles $\phi_{1}$ and $\phi_{2}$. Because $\phi_{1} \phi_{2}$ can couple to the right-handed neutrinos via the dimension-five operators suppressed by the GUT scale $M_{G U T}$, DM particle has a natural lifetime around $\tau \sim 10^{26} \mathrm{~s}$ if the seesaw scale is about $10^{4} \mathrm{GeV}$. In particular, the DM particle will decay dominantly to the $\mu$ and $\tau$ final states due to the $N_{2}$ dominant seesaw mechanism. Moreover, the DM relic density, which usually is a problem in decaying DM models, can be achieved naturally through the freeze-in mechanism with couplings typically 
about $\mathcal{O}(\mathrm{TeV}) / M_{G U T}$. Simultaneously the small scale problem on the power spectrum can be solved since the metastable particles in the DM sector, which are also freezed into the thermal bath, can decay to the relativistic LSP in the supersymmetric SMs. Furthermore, we showed that the baryon asymmetry can be generated via the soft leptogenesis in a large region of the parameter space for supersymmetry breaking soft mass terms.

\section{Acknowledgments}

We would like to thank S. Matsumoto for helpful discussions. This research was supported in part by the DOE grant DE-FG03-95-Er-40917 (TL), by the Natural Science Foundation of China under grant No. 10821504, and by the Mitchell-Heep Chair in High Energy Physics (TL).

\section{Appendix A: A Brief Review of Freeze-In Mechanism}

In this Appendix we will give a brief review of the freeze-in mechanism, but we shall formulate it differently. The basic idea of freeze-in mechanism is that in the BE for FIMP $X$, there is no inverse decay or scattering process to the mother particle that produces FIMP, as a result even small interaction rate is also successful in generating significant relic density for FIMP. As a starting point, the simple BE for $X$ is (We consider the scattering process as an example, and the similar expression holds for decay.)

$$
Y^{\prime}(z)=\frac{s z}{H_{1}} \frac{\gamma(A+B \rightarrow X+C)}{\left(s Y_{A}^{e q}\right)\left(s Y_{B}^{e q}\right)} Y_{A}(z) Y_{B}(z),
$$

where the Hubble constant at $T=M_{A}$ is $\left.H_{1}(T)\right|_{M_{A}}=1.66 \sqrt{g_{*}^{\rho}} T^{2} /\left.M_{p l}\right|_{M_{A}}$. In this paper, we use $g_{*}^{\rho, s}$ to denote the effective numbers of degree of freedom in the thermal bath at the freeze-in temperature $T \sim M_{A}$, respectively for the entropy density $s$ and energy density $\rho$.

If $A$ and $B$ are assumed to be in thermal equilibrium during freeze-in, the $\mathrm{BE}$ reduces to the situation discussed in Ref. 14]. The FIMP is produced dominantly at the temperature around the mass of heavier bath particles, when the bath particles still track their equilibrium distribution closely [14]. So the approximations are valid. In our paper, from Eq. (41) one finds that $\widetilde{N}$ and $N$ deviate from their equilibrium typically at $T_{f} \sim M_{N} / 5$ (due to strong washout, sRHNs departure from equilibrium rather late), so the equilibrium approximation is also employed here.

In our model, FIMP is freezed-in both from $N_{ \pm}$decays and their scatterings. First let us discus the decay. The yield of $X$ is produced simply by integrating the right-hand side of Eq. (A1) over $z$ from 0 to $\infty$. A good property of freeze-in mechanism is that $Y(x)$ 
is insensitive to the UV physics, which is obvious from the integrand. So one can safely ignore the time of lower bound which may sensitive to inflation or reheating at the UV. For two-body decay, the thermally averaged decay rate is easily obtained analytically

$$
\gamma(A \rightarrow X+C)=\frac{g_{A} T^{3}}{2 \pi^{2}} z^{2} K_{1}(z) \Gamma(A \rightarrow X+C),
$$

with $g_{A}$ the internal degrees of freedom of $A$. Furthermore, let us reasonably assume that the freeze-in process lasts from $z \approx 0$ till $z \gtrsim \mathcal{O}(1)$ when generally the weakly interacting particle $A$ decouples from thermal bath. Then one has

$$
\begin{aligned}
Y(z \gtrsim 10) & \approx \int_{0}^{\infty} d z \frac{z}{s H_{1}} \gamma(A \rightarrow X+C) \\
& =\frac{135 g_{A}}{8 \pi^{3}(1.66) g_{*}^{s} \sqrt{g_{*}^{\rho}}} \frac{M_{p l} \Gamma(A \rightarrow X+C)}{M_{A}^{2}} .
\end{aligned}
$$

Then the relic density of $X$ is given by

$$
\Omega_{X} h^{2} \approx 4.50 \times 10^{25} \times \lambda^{2} \frac{g_{A}}{g_{*}^{s} \sqrt{g_{*}^{\rho}}} \frac{M_{X}}{M_{A}},
$$

where we have typically used $\Gamma(A \rightarrow X+C)=\lambda^{2} M_{A} / 8 \pi$ and dropped the phase space factor. If multi thermal particles $A_{i}$ contribute to freeze-in, $i$ should be summed over. Next we study the freeze-in mechanism through scattering processes. The thermally averaged scattering rate is formally given by

$$
\begin{aligned}
\gamma(A+B \rightarrow X+C) & =\frac{g_{A} g_{B} T^{6}}{16 \pi^{4}} \int_{\left(m_{A}+m_{B}\right)^{2} / s}^{\infty} d x x^{4} K_{1}(x) \lambda\left(1, x_{A}, x_{B}\right) \\
& \times \sigma(A+B \rightarrow X+C),
\end{aligned}
$$

where $x=\sqrt{s} / T$, and then the integrand depends on $z$ through its $s$ dependence. Similarly, the final yield of $X$ is obtained by integrating over $z$ from 0 to some large value

$$
\begin{aligned}
Y_{X}(\infty) & \approx \int_{0}^{\infty} d z \frac{z}{s H_{1}} \gamma(A+B \rightarrow X+C) \\
& =\frac{45 g_{A} g_{B}}{2 \times 1.66 \times 256 \pi^{7} g_{*}^{s} \sqrt{g_{*}^{\rho}}} \frac{M_{p l}}{M_{A}} \times \mathcal{I}[x, z], \\
\mathcal{I}[x, z] & \equiv \int_{0}^{\infty} d z \int_{z}^{\infty} d x x^{2} K_{1}(x) \Xi(x, z) \sim \mathcal{O}(1),
\end{aligned}
$$

where $\Xi(x, z)=(16 \pi s) \lambda\left(1, x_{A}, x_{B}\right) \sigma(x, z)$, and $\sigma(x, z)$ is the scattering cross section. Eventually, the relic density is

$$
\Omega_{X} h^{2} \approx 6.0 \times 10^{22} \times \frac{g_{A} g_{B}}{g_{*}^{s} \sqrt{g_{*}^{\rho}}} \times\left(\frac{M_{X}}{M_{A}}\right) \times \mathcal{I}[x, z] .
$$


[1] O. Adriani et al. [PAMELA Collaboration], Nature 458, 607 (2009) arXiv:0810.4995 [astro$\mathrm{ph}]$.

[2] A. A. Abdo et al. [The Fermi LAT Collaboration], Phys. Rev. Lett. 102, 181101 (2009) arXiv:0905.0025 [astro-ph.HE]].

[3] N. Arkani-Hamed, D. P. Finkbeiner, T. R. Slatyer and N. Weiner, Phys. Rev. D 79, 015014 (2009); M. Cirelli, R. Franceschini and A. Strumia, Nucl. Phys. B 800, 204 (2008); M. Ibe, H. Murayama and T. T. Yanagida, Phys. Rev. D 79, 095009 (2009); W. L. Guo and Y. L. Wu, Phys. Rev. D 79, 055012 (2009).

[4] F. Aharonian et al. [H.E.S.S. Collaboration], Phys. Rev. Lett. 101, 261104 (2008) arXiv:0811.3894 [astro-ph]].

[5] F. Aharonian et al. [H.E.S.S. Collaboration], Astron. Astrophys. 508, 561 (2009) arXiv:0905.0105 [astro-ph.HE]].

[6] J. Liu, Q. Yuan, X. Bi, H. Li and X. Zhang, Phys. Rev. D 81, 023516 (2010) arXiv:0906.3858 [astro-ph.CO]]. Y. Nomura and J. Thaler, Phys. Rev. D 79, 075008 (2009) arXiv:0810.5397 [hep-ph].

[7] A. Ibarra and D. Tran, JCAP 0807, 002 (2008) arXiv:0804.4596 [astro-ph]]. K. Ishiwata, S. Matsumoto and T. Moroi, Phys. Rev. D 78, 063505 (2008) arXiv:0805.1133 [hep-ph]]. K. Ishiwata, S. Matsumoto and T. Moroi, Phys. Lett. B 675, 446 (2009) arXiv:0811.0250 [hep-ph]].

[8] A. Arvanitaki, S. Dimopoulos, S. Dubovsky, P. W. Graham, R. Harnik and S. Rajendran, Phys. Rev. D 79, 105022 (2009) arXiv:0812.2075 [hep-ph].

[9] P. f. Yin, Q. Yuan, J. Liu, J. Zhang, X. j. Bi and S. h. Zhu, Phys. Rev. D 79, 023512 (2009) arXiv:0811.0176 [hep-ph]]. E. Nardi, F. Sannino and A. Strumia, JCAP 0901, 043 (2009) arXiv:0811.4153 [hep-ph]. J. T. Ruderman and T. Volansky, arXiv:0907.4373 [hep-ph]. A. Ibarra and D. Tran, JCAP 0902, 021 (2009) arXiv:0811.1555 [hep-ph].

[10] X. Gao, Z. Kang and T. Li, arXiv:1001.3278 [hep-ph].

[11] X. J. Bi, P. H. Gu, T. Li and X. Zhang, JHEP 0904, 103 (2009) arXiv:0901.0176 [hepph]]. I. Gogoladze, N. Okada and Q. Shafi, Phys. Lett. B 679, 237 (2009) arXiv:0904.2201 [hep-ph]]. P. H. Gu, H. J. He, U. Sarkar and X. m. Zhang, Phys. Rev. D 80, 053004 (2009) arXiv:0906.0442 [hep-ph]]. C. H. Chen, arXiv:0905.3425 [hep-ph]. C. H. Chen, C. Q. Geng and D. V. Zhuridov, JCAP 0910, 001 (2009) [arXiv:0906.1646 [hep-ph]].

[12] S. F. King, JHEP 0508, 105 (2005) arXiv:hep-ph/0506297]. S. F. King, Nucl. Phys. B 562, 
57 (1999) arXiv:hep-ph/9904210.

[13] X. J. Bi, R. Brandenberger, P. Gondolo, T. j. Li, Q. Yuan and X. m. Zhang, Phys. Rev. D 80, 103502 (2009) [arXiv:0905.1253 [hep-ph]].

[14] L. J. Hall, K. Jedamzik, J. March-Russell and S. M. West, arXiv:0911.1120 [hep-ph].

[15] W. B. Lin, D. H. Huang, X. Zhang and R. H. Brandenberger, Phys. Rev. Lett. 86, 954 (2001) arXiv:astro-ph/0009003.

[16] M. Fukugita and T. Yanagida, Phys. Lett. B 174, 45 (1986).

[17] G. D'Ambrosio, G. F. Giudice and M. Raidal, Phys. Lett. B 575, 75 (2003) arXiv:hep-ph/0308031.

[18] Y. Grossman, T. Kashti, Y. Nir and E. Roulet, Phys. Rev. Lett. 91, 251801 (2003) arXiv:hep-ph/0307081.

[19] S. Matsumoto and K. Yoshioka, arXiv:1006.1688 [hep-ph].

[20] G. Altarelli and F. Feruglio, Nucl. Phys. B 720, 64 (2005) arXiv:hep-ph/0504165.

[21] P. Meade, M. Papucci, A. Strumia and T. Volansky, arXiv:0905.0480 [hep-ph].

[22] T. Fukuyama and H. Nishiura, arXiv:hep-ph/9702253.

[23] P. F. Harrison, D. H. Perkins and W. G. Scott, Phys. Lett. B 530, 167 (2002) arXiv:hep-ph/0202074. P. F. Harrison and W. G. Scott, Phys. Lett. B 535, 163 (2002) arXiv:hep-ph/0203209. Phys. Lett. B 557, 76 (2003) arXiv:hep-ph/0302025.

[24] I. de Medeiros Varzielas, S. F. King and G. G. Ross, Phys. Lett. B 644, 153 (2007) arXiv:hep-ph/0512313.

[25] T. Schwetz, M. A. Tortola and J. W. F. Valle, New J. Phys. 10, 113011 (2008) arXiv:0808.2016 [hep-ph]]. G. L. Fogli, E. Lisi, A. Marrone, A. Palazzo and A. M. Rotunno, Phys. Rev. Lett. 101, 141801 (2008) arXiv:0806.2649 [hep-ph]].

[26] S. R. Klein [IceCube Collaboration], IEEE Trans. Nulc. Sci. 56, 1141 (2009) arXiv:0807.0034 [astro-ph]].

[27] M. R. Buckley, K. Freese, D. Hooper, D. Spolyar and H. Murayama, Phys. Rev. D 81, 016006 (2010) arXiv:0907.2385 [astro-ph.HE]].

[28] P. Colin, V. Avila-Reese and O. Valenzuela, Astrophys. J. 542, 622 (2000) arXiv:astro-ph/0004115.

[29] S. P. Martin, arXiv:hep-ph/9709356.

[30] G. F. Giudice, L. Mether, A. Riotto and F. Riva, Phys. Lett. B 664, 21 (2008) arXiv:0804.0166 [hep-ph]].

[31] S. Davidson, E. Nardi and Y. Nir, Phys. Rept. 466, 105 (2008) arXiv:0802.2962 [hep-ph]].

[32] Y. Grossman, T. Kashti, Y. Nir and E. Roulet, JHEP 0411, 080 (2004) 
arXiv:hep-ph/0407063.

[33] C. S. Fong and M. C. Gonzalez-Garcia, JHEP 0903, 073 (2009) [arXiv:0901.0008 [hep-ph]].

[34] R. Barbieri, P. Creminelli, A. Strumia and N. Tetradis, Nucl. Phys. B 575, 61 (2000) arXiv:hep-ph/9911315.

[35] E. Nardi, Y. Nir, E. Roulet and J. Racker, JHEP 0601, 164 (2006) arXiv:hep-ph/0601084.

[36] S. Antusch, S. F. King and A. Riotto, JCAP 0611, 011 (2006) arXiv:hep-ph/0609038.

[37] E. Komatsu et al. [WMAP Collaboration], Astrophys. J. Suppl. 180, 330 (2009) arXiv:0803.0547 [astro-ph]]. 